\title{
“Estadio de excepción": reflexiones sobre el Estado de Excepción a propósito de los mundiales de fútbol profesional
}

\section{"State-dium of Emergency". Reflections on State of Emergency in relation with FIFA World Cups}

\author{
Ricardo Cuevas Bascuñán' \\ Universidad de Valparaíso, Chile
}

Resumen

El pasado Mundial de Fútbol organizado por la FIFA en Brasil dejó en evidencia no solo en lo que se ha transformado el deporte hoy en día, sino también, el tipo de sociedad en la cual vivimos y sus correspondientes Estados, es decir, una sociedad de control amparada en un Estado de Excepción constante. Un ejemplo particular resalta esta tesis: el caso de los aficionados chilenos, quienes ingresaron sin boletos y por el sector de prensa al Estadio Maracaná, quienes posteriormente fueron expulsados del país. A este caso es posible sumar un antecedente de similares características en cuanto a la solución adoptada: cuando barrabravas argentinos fueron expulsados de Sudáfrica en el Mundial de Fútbol FIFA de dicho país. Sin embargo, los casos de Brasil y Sudáfrica no son aislados, ni siquiera unas instancias limítrofes relativas a este magno-evento, sino más bien la generalidad dentro de un espectáculo cada vez más intervenido, tanto por el Estado como por los organismos de seguridad (pública y privada).

Palabras clave: Agamben; Chile; estado de excepción, fútbol profesional; mundial de Brasil.

\begin{abstract}
The last World Cup by FIFA in Brazil made evident not only in what has become the sport today, but also the type of society in which we live and their corresponding states, i. e.: a society control covered in a state of constant exception. A particular case highlights this thesis: the Chilean fans who entered without tickets by the Maracana Stadium's press room. Then, they were subsequently deported. Add to that, a history of similar characteristics in terms of the solution adopted: when Argentine hooligans were expelled from South Africa during the FIFA World Cup in the country.

However, the cases of Brazil and South Africa are not isolated, even extremely facts of this great-event Rather this is a generality of an increasingly intervened show, both from the State and agencies security (public and private).
\end{abstract}

Keywords: Agamben; Chile; state of emergency, professional football; world cup in Brazil.

Recibido: 21 de noviembre de 2014

Aceptado: 18 de diciembre de 2014

\section{Introducción}

El reciente Mundial de Fútbol FIFA realizado en Brasil nos dejó un buen número de acontecimientos y sucesos para cuestionar, reflexionar y comprender desde las ciencias sociales, empezando por el espectáculo como tal, hasta el contexto y coyuntura bajo el cual este se desarrolló.

Desde este punto de vista el presente ensayo tiene como propósito cuestionar, ya no solo qué

1 Egresado de Sociología por el Instituto de Sociología de la Facultad de Humanidades, Universidad de Valparaíso (Valparaíso, Chile). Contacto: rcuevasbascunan@gmail.com 
es lo que acontece y quiénes son sus principales actores, sino más bien, comprender cómo y por qué sucedieron determinadas acciones. De allí que tomemos como referencias dos acontecimientos que, sin dudas, marcaron la historia, para bien o para mal, de los mundiales de fútbol: la expulsión de los barrabravas argentinos de Sudáfrica 2010 en el marco del partido de la selección albiceleste contra Nigeria, y la expulsión de los hinchas chilenos de Brasil, luego del partido de Chile versus España.

Más allá del repudio internacional manifestado por los medios de comunicación y de la gravedad de los castigos que afectaron a los involucrados, resulta interesante cuestionarel ¿hasta dóndellega el poder de la FIFA en cuanto a políticas de sanción y disciplina?, o mejor aún, ¿por qué los gobiernos, en ambos casos, determinaron la expulsión de los individuos, y si hubiesen sancionado de la misma forma si los acontecimientos ocurrieran en otro tipo de espectáculo?

Sin dudas, situar al fútbol profesional dentro de un Estado de Excepción puede resultar controversial, inaceptable para algunos y difícil de asimilar para otros. Sin embargo, quienes asisten cotidianamente como espectadores a partidos de fútbol profesional, lograrán comprobar cómo ciertas prácticas no se corresponden con un Estado democrático, si se consideran las respectivas diferencias socioculturales entre países.

Entonces, es necesario, con el fin de comprender las dinámicas del fútbol actual, encontrar si la FIFA y sus afiliadas nacionales (asociaciones de fútbol respectivas a cada país) son agencias que, más allá de ser órganos rectores o entes disciplinarios exclusivos del fútbol, resultan ser actores principales en la discreción de las políticas de gobierno. Aquello muestra que tal incursión en la discrecionalidad les permiten adoptar un carácter de exclusividad y abstracción de la ley misma; en palabras del filósofo Giorgio Agamben: el Estado de Excepción en su máxima expresión, la aplicación de la ley estando fuera de ella (Agamben, 2010).
Una de las máximas que rige los Estados de Excepción es considerar a los individuos en general como potenciales enemigos: cualquiera puede ser un terrorista o transgresor que busca desestabilizar la soberanía, las instituciones o cualquier tipo de organismo que funcione como agencia. En el caso del fútbol, considerar al espectador como un sujeto potencialmente riesgoso no es una situación nueva, y siempre que se ha necesitado considerarlo de esta forma se construyen tipologías que lo identifican colectivamente En Inglaterra se les llamó hooligans, en el resto de Europa, ultras, en Latinoamérica, barras bravas y torcidas; grupos que, además de ser señalados por su carácter violento y delictual, no se tardó en asociarlos al consumo excesivo de alcohol y venta drogas.

En el marco de este planteamiento, y a la luz ded los casos de estudio, se suma la política estatal enfocada a los asitentes del fútbol profesional chileno, a saber: la implementación del Plan Estadio Seguro, iniciado durante la gestión del ex presidente Sebastián Piñera, y continuado hasta el día de hoy en el gobierno de Michelle Bachelet.

Comprender el Estado de Excepción como paradigma del Estado moderno es entender cómo su funcionamiento biopolítico se desarrolla en los espacios que históricamente han sido considerados como apolíticos 0 despolitizados, como lo son los ambientes de ocio y recreación. El fútbol profesional, lejos de ser una instancia-espectáculo apolítica, ha demostrado ser vital para la subsistencia de este tipo de Estado.

\section{De sudáfrica 2010 a brasil 2014}

\section{Los barrabravas argentinos en Sudáfrica}

Uno de los principales antecedentes más inmediatos lo podemos situar en el Mundial de Fútbol de 2010 organizado en Sudáfrica, donde por primera vez se toma una medida de Estado a propósito de actividades ilegales relacionadas con el fútbol: 
Sudáfrica deportará a 17 barrabravas argentinos luego de detenerlos por acusarlos de ocasionar disturbios durante el partido entre Argentina y Nigeria, informó la policía [...] Con éstos ya suman unos 30 los simpatizantes de Argentina que fueron obligados a regresar a su país por problemas de comportamiento o antecedentes policiales (BBC Mundo, 2010).

A partir de este caso podemos identificar una serie de situaciones que acontecen y generan problemas de carácter político, social y cultural, tanto para los organizadores como para el Estado sudafricano. De estos enunciaremos algunos, y solo profundizaremos en aquellos más importantes para la presente reflexión.

En primera instancia, y sobre todo para el caso argentino, queda en evidencia la fuerte relación entre barristas y dirigentes de la Asociación del Fútbol Argentino (AFA), e incluso actores políticos de importancia.

Los argentinos deportados fueron detenidos en la escuela de Pretoria donde se alojan los integrantes de la ONG Hinchadas Unidas Argentinas, que trajo más de 200 "barras" a Sudáfrica para alentar a la selección argentina en el Mundial [...] Esta agrupación, que nuclea a hinchas de una treintena de clubes de fútbol, es liderada por el dirigente Marcelo Mallo, vinculado al movimiento político del ex presidente argentino Néstor Kirchner (20032007), esposo de la actual mandataria Cristina Fernández (La Tercera, 2010).

En segunda instancia, el "problema" ocasionado por las facilidades que tuvieron estos para salir del país de origen, donde el control aduanero no los consideró como sujetos de peligro, permitiendo la salida de aquellos que incluso se encontraban con libertad condicional: "Al menos dos de esos individuos son líderes de barrabravas de clubes y un tercero está en libertad condicional por asesinato, informó un comunicado de la policía sudafricana" (EI Universo, 2010). Mientras que otra publicación virtual señaló un dato más importante aún de uno de los barristas expulsados:
En el grupo se encontraba Ariel Pugliese, alias 'El Gusano', un ex líder de la barrabrava de Nueva Chicago, equipo del fútbol de ascenso, investigado en 2007 por el homicidio de un hincha del rival Tigre, y que fuera puesto por la AFA como guardaespaldas de Lionel Messi en uno de sus pasos por Buenos Aires (ZGS, 2010).

Por parte de los barristas, luego de su detención y posterior expulsión, no dudaron en señalar a altos dirigentes como financistas y colaboradores, incluso nombrando al entonces director técnico del seleccionado albiceleste, Diego Armando Maradona.

Maradona desmintió las acusaciones el martes en Pretoria, cuando el escándalo había estallado en Buenos Aires. "No tuve ninguna relación con ningún personaje. $Y$ si ese personaje dice que yo lo traje acá. Y si quiere fama, que vaya a laburar (trabajar). Acá no entra nadie ni va a entrar (a la concentración argentina)", respondió el DT (ZGS, 2010).

Ahora bien, como tercer punto, menos evidente que los anteriores, observamos las diferencias en los criterios entre federaciones de fútbol, tanto en el nivel central (FIFA) como en el descentralizado (en este caso la AFA), en especial, el del tipo de espectador permitido. En Europa, continente donde se ha establecido históricamente la sede de la FIFA, se ha implementado un sistema de control en los accesos y de restricción en el derecho de admisión, principalmente, a partir de la implementación del Plan Taylor en Inglaterra durante el mandato de Margaret Thatcher. Sin embargo, estos controles no fueron aplicados ni ejercidos en el Mundial de Sudáfrica, solo a partir de los incidentes ocurridos previos al partido entre las selecciones de Nigeria y Corea del Norte (avalancha humana que dejó 16 heridos), se puso en ejercicio una política de tolerancia cero contra aquellos considerados como los "hinchas violentos del fútbol".

Queda en evidencia que no son solo las diferencias culturales las que separan al continente europeo con el americano en cuanto 
al fútbol profesional, sino también el desarrollo del espectáculo dependerá directamente de los tipos de gobierno nacionales, particularmente en su interacción con el mercado (entendiendo este como un ente supranacional, de quien dependen las economías nacionales).

Como cuartoy último punto, podemos considerar la necesidad de los países considerados del tercer mundo, de ser no solo partícipes, sino también organizadores y buenos anfitriones, de los espectáculos considerados ya del primer mundo. Con esto nos referimos al fútbol, obviando su carácter deportivo; es decir, el fútbol como mercancía y como institución. En palabras del presidente de la FIFA, Joseph Blatter:

Se puede sentir eso por todas partes, ustedes (los periodistas) también, espero que este Mundial sea muy especial, el primero sobre el continente africano. Va a probar que el continente africano y Sudáfrica son capaces de organizar tal evento (El Universo, 2010).

\section{El período entre Mundiales: Chile y el Plan Estadio seguro}

En Chile las barras emprenden su crecimiento a finales de la década de los ochenta, en concordancia con el ocaso de la dictadura militar, siendo la primera en formarse la barra del club deportivo Colo-Colo, seguida por la de Universidad de Chile, la Universidad Católica, la de Santiago Wanderers, entre las más numerosas. Si bien es posible observar ciertas situaciones delictuales durante la década de los noventa, es a comienzos del siglo XXI cuando la opinión pública y los medios de comunicación le dan una cobertura mayor, destacando el enfoque casi criminal de estas asociaciones o agrupaciones.

La privatización del fútbol profesional contribuyó a una reestructuración en las relaciones entre dirigentes deportivos y líderes de barras, donde la primera figura organizacional que vio la luz fueron las sociedades anónimas deportivas (SAD). Sus principales representantes eran accionistas con un alto poder de injerencia política (bien desde dentro o desde fuera del establecimiento), como es el caso del diputado Gustavo Hasbún y el presidente de la República Sebastián Piñera (figuras administrativas del Colo-Colo), o José Yuraszeck (empresario considerado como uno de los más ricos de Chile, con asiento en Universidad de Chile).

Cuando fueron más evidentes, los gobiernos pertenecientes a la Concertación de Partidos por la Democracia no abordaron el problema de las barras como un asunto nacional, ni siquiera como de Estado. Sus comportamientos delictuales, si bien preocupaban, eran dejados en manos del Poder Judicial. Sin embargo, esta situación cambia radicalmente con la llegada de la derecha al poder: durante el gobierno del presidente Sebastián Piñera se comienza a hablar del "problema de las barras bravas"; así, se adopta una política de tolerancia cero y de persecución a quienes se vean involucrados en hechos de violencia relacionada con el deporte.

La postura del gobierno se traduce en un plan destinado a "normalizar"la conducta del público asistente, en resguardo de su propia seguridad, bajo el eslogan "que vuelva la familia a los estadios". Este proyecto comienza a gestarse durante el año 2011, siendo 2012 el año de su puesta en escena, con el principal objetivo de convertirse en una política pública nacional.

Este programa es una de las primeras medidas aplicadas directamente a las barras en Latinoamérica con características tan similares a las del gobierno autoritario de Margaret Thatcher. Dicho plan tiene como principal gestor al Ministerio del Interior y Seguridad Pública, en coordinación con las Intendencias Regionales y las Fuerzas de Orden y Seguridad Pública (Carabineros de Chile y sus unidades de Fuerzas Especiales).

Como primer punto debemos contemplar cuáles son las características principales de este plan, para vislumbrar de mejor forma sus falencias o las posibles correcciones. En la Cuenta Pública Sectorial del Ministerio del Interior y Seguridad Pública (Gobierno de Chile, 2012) se expuso lo 
siguiente en cuanto los lineamientos de este programa:

- Gestión: coordinación con Intendencias y Carabineros de Chile en la preparación de los espectáculos deportivos, especialmente aquellos calificados de alto riesgo.

- Marco legal: se modifica la Ley 19.327, la cual contiene normas para la prevención y sanción de hecho de violencia en recintos deportivos con ocasión de espectáculos de fútbol profesional (Boletín 4864-29). Respecto a estas indicaciones, cabe resaltar que incluyen un nuevo catálogo de faltas y sanciones, nuevas obligaciones para los organizadores de espectáculos de fútbol profesional y para los intendentes. Asimismo, en cuanto a los organizadores de espectáculos de fútbol profesional, se les exige que los recintos deportivos destinados a los referidos espectáculos cuenten con sistemas de control de acceso y de grabación de los asistentes. Por otro lado, la indicación elimina el concepto de "partidos de alto riesgo", ya que se considera que se deberán tomar medidas de acuerdo con el riesgo asociado al evento deportivo que se realice. Finalmente, se elimina el concepto de "barra" de la ley.

- Infraestructura: se realizó un estudio donde se definieron criterios generales para implementar sistemas de control de acceso a los espectáculos de fútbol profesional.

- Judicial: desde la implementación del plan Estadio Seguro se han presentado 99 querellas, con un total de 156 imputados a quienes se les ha aplicado la Ley de Violencia en los Estadios.

La persecución de ciertas conductas, como la violencia, empieza a involucrar a todos aquellos que alguna vez hayan tenido participación o vinculación en algún delito o infracción de esa índole. Esto si tomamos la exclusión manifiesta que se desea efectuar y aplicar por parte del Estado. Sin embargo, también es posible apreciar una exclusión colateral, que se evidencia en el alejamiento de las familias, debido a los tratos vejatorios a las que se ven expuestas por parte de las fuerzas policiales al intensificar su búsqueda de objetos o vigilar conductas que puedan ser catalogadas como peligrosas.

Este dispositivo de seguridad exacerbado se puede interpretar como un procedimiento ad hoc en un contexto político-administrativo que se torna de carácter autoritario, con un alto nivel represivo; es decir, el aumento de poder a los efectivos policiales (similar a la de una dictadura) no necesariamente asegura una disminución de la violencia (punto principal a erradicar). Así, por ejemplo, ha sido evidente que los Carabineros de Chile tiene una orientación disuasiva, pero no persuasiva, por lo que, ante la existencia de conflictos latentes, la solución más rápida y efectiva distará de ser del todo pacífica.

Quienes elaboran estas políticas suelen ser partidarios de estas técnicas porque es relativamente fácil añadirlas a las ya existentes y porque tranquilizan a los ciudadanos, al darles la impresión de que se están tomando medidas contundentes contra la criminalidad. Sin embargo, como tales medidas no se interesan en las causas subyacentes a ésta -como son las desigualdades sociales, el desempleo y la pobreza-, sus éxitos principales radican en la protección frente a la delincuencia de ciertos grupos de la población $y$ en el desplazamiento de ésta hacia otros ámbitos (Gidddens, 2001: 289).

La eliminación del concepto de barra fue negar y prohibir nuevas formas de agrupación y de asociación, derecho garantizado por el Estado siempre y cuando no tengan una orientación delictual manifiesta, es decir, mientras no se compruebe que las barras son agrupaciones criminales, no se puede prohibir su conformación. Las barras, como se mencionó, 
responden a nuevas cosmovisiones del deporte, contemplando la formación de nuevas identidades, con características culturales propias, pero similares a las antiguas formas de representación social. Se busca criminalizar e ilegalizar a las colectividades asociadas con el fútbol: en el momento que se habla de familias, se refieren específicamente a familias individualizadas, grupos familiares que no se relacionen entre sí.

La efectividad del plan solo se ha visto en el número de detenidos por partido de fútbol (un mero análisis estadístico), mientras que las familias, principales beneficiarias de este programa, no han vuelto al estadio, o no en la medida esperada. El fútbol está dejando de ser un espectáculo: se está minando lo relativo con el folklore extradeportivo, al buscar un disciplinamiento de la conducta que busca una igualación del público asistente a los recintos de fútbol con el del resto de espectadores de otros deportes, como el caso del tenis o del golf, donde una de sus características principales es su actitud calmada y silente. En contraste, los seguidores del fútbol se caracterizan por su efusividad o, como ellos dicen, por la "pasión" con la que viven el deporte.

Otra falencia del programa es que, si bien se criminaliza y estigmatiza a los barristas, buscando que vuelva la familia a los estadios, con el objetivo consecuente de atraer a más gente a los recintos deportivos, resulta desdibujado, debido que la mayoría de los asistentes a los partidos de fútbol son barristas; en contraste con la asistencia de socios, accionistas o "familias", que en número no logra ni igualarlos. Otra política ha sido el incremento del valor de las entradas, lo cual no necesariamente asegura la inasistencia de delincuentes, pero sí impacta en la cada vez menos asistencia de las familias, sobre todo de aquellas que pertenecen a estratos socioeconómicos bajos. Si bien el gobierno no tiene injerencia directa en el los precios, sino que este corre por parte de sus organizadores, accionistas y dirigentes deportivos, su relación es indirecta.

\section{Copa del Mundo Brasil 2014 y la expulsión de la Marea Roja}

Existe un antiguo refrán que dice que de los errores se aprende, y quienes dominan el fútbol lo han sabido aplicar. Las experiencias en Sudáfrica 2010 y en Chile desde el 2011 han dado cuenta de ello: la FIFA dejó de experimentar y ejerció de lleno el nuevo y mejorado plan de acción.

En una primera fase, varios meses antes de la realización del Mundial, mediante la web se puso a disposición global los boletos de entrada, a valores muy superiores, en comparación con el certamen de cuatro años atrás. En este punto podemos resaltar que, si bien la disposición fue "abierta", es necesario comprender que aquellos interesados en asistir debían contar, en primera instancia, con una buena conectividad a internet (primer filtro de exclusión); como segundo punto, se debía contar con tarjetas de crédito o débito, debido a este sistema de compra (segundo filtro de exclusión); y tercero, contar con un poder adquisitivo considerable, debido al altísimo valor de las entradas. No obstante las promociones de entrada a varios partidos, los precios ahuyentaban a un público del tercer mundo, como es considerado el público latinoamericano (tercer filtro de exclusión).

En una segunda fase, una vez iniciado el torneo, observamos el "aprendizaje" de la FIFA en acción. El contexto en el cual se desarrollaría el espectáculo no era el mejor para sus organizadores: la gran cantidad de protestas sociales en las principales urbes del país ponía en jaque la efectividad de los dispositivos de seguridad; sin embargo, estos respondieron de forma eficiente, logrando que las voces de disidencia se mantuviesen alejadas de los recintos. Solo hubo un momento en que el malestar social brasilero se hizo evidente, cuando durante la ceremonia de inauguración, en el momento en que se nombra a la presidenta Dilma Rousseff y al presidente de la FIFA Joseph Blatter, fueron duramente insultados, situación 
que en la Copa Confederaciones ya había acontecido: una gran silbatina se "tomó" el Estadio Nacional de Brasilia, en un claro gesto de reprobación de la gestión de ambos.

Bajo estruendosos gritos de desaprobación, Blatter declaró que la FIFA y los aficionados "están todos unidos para una fiesta del fútbol en el país pentacampeón" y expresó su "placer" por darle la "bienvenida" al estadio a "las autoridades brasileñas y a la presidenta Dilma Rousseff". El abucheo no cesaba y el timonel del organismo rector del fútbol increpó al público: "Amigos brasileños, ¿dónde está el respeto al fair play?", tras lo cual pasó la palabra a Rousseff, quien no disimulaba su malestar (Radio Cooperativa, 2013).

Sin embargo, a pesar de los efectivos que resultaron los dispositivos de seguridad, antes del inicio del encuentro entre los seleccionados nacionales de Chile y España, 85 hinchas chilenos son detenidos por su entrada ilícita al Estadio Maracaná. Este hecho que sin dudas marcó el lado extrafutbolístico del Mundial, generando un impacto mediático instantáneo y de magnitud global. En un confuso incidente los barristas lograron entrar por accesos no destinados al público, sin embargo, solo pudieron llegar hasta la sala de prensa, donde fueron retenidos para posteriormente culminar su detención en una comisaría local.

"Igual la hicimos". Así resumió el incidente uno de los 85 hinchas chilenos detenidos por intentar entrar al partido Chile-España sin pagar la entrada. Frente a los micrófonos y cámaras de la prensa explicó -con cierta satisfacción personal- que "uno ve la oportunidad y entramos... se hizo tira dos puertas y se cayó un panel, nada más" (Fernández, 2014).

La FIFA no dudó en condenar rápidamente el suceso, apelando a la extrema violencia que usaron los barristas como excusa ante las falencias evidenciadas en los dispositivos de seguridad. Por su parte, el gobierno brasilero, sumándose a la reprobación generalizada, adoptó como medida excepcional, la expulsión del país de todas aquellas personas detenidas por incidentes vinculados al Mundial.

Acoso sexual, invasión al estadio, vandalismo, robo y falsificación. Esos son los cargos que registra la hinchada local en las dos primeras semanas de Brasil 2014. Una parte de la fanaticada chilena ha 'deslucido' en Brasil. No son los únicos. La hinchada argentina también suma incidentes (Fernández, 2014), los cuales tuvieron su réplica en Chile, donde el presidente de la Asociación Nacional de Fútbol Profesional (ANFP), Sergio Jadue, anunció que los detenidos y expulsados serían sancionados.

Considerando los graves hechos protagonizados por un grupo de chilenos en el Estadio Maracaná de Río de Janeiro el pasado 18 de junio, con ocasión del encuentro por la Copa del Mundo entre España y Chile, y por instrucción del Presidente de nuestra Federación, don Sergio Jadue, se coordinó con el jefe del Plan Estadio Seguro (José Roa) a fin de obtener los antecedentes de parte de las autoridades respectivas sobre los motivos para deportar a estas personas de Brasil. Una vez que se reciba la información, se procederá a aplicar a los involucrados la Ley de la República de Chile N 19.327, sobre Prevención y Sanción de hechos de Violencia en los Estadios, para de esa manera impedirles el ingreso a los recintos deportivos donde se juegue fútbol profesional en nuestro país (González, 2014).

Teniendo en cuenta tales medidas, resulta primordial cuestionar la real magnitud que ha logrado el fútbol, especialmente los costos sociales y políticos que implica quebrantar las normas extrafutbolísticas que lo regulan. Las sanciones para los hooligans fueron penas de treinta años de presidio, considerando al hooliganismo como un delito grave, similar a un homicidio o una violación sexual. Los barristas que hoy en día delincan en el extranjero son expulsados, no solo de los recintos deportivos, sino también de las naciones, además de ser castigados con la prohibición de entrada a este 
tipo de eventos. Lo que ha servido como excusa perfecta para incrementar los "requisitos" a cumplir para poder ingresar a los estadios de fútbol.

\section{El fútbol como pilar de la sociedad de control}

En primera instancia, resulta pertinente entender el contexto social en el cual situamos nuestro análisis, el por qué nos asentamos en el marco epistémico del Estado de Excepción, como paso previo a vincularlo con los casos concretos a analizar.

A partir del análisis realizado por Foucault durante el sigloXX, la Sociedad Disciplinaria, en su máxima expresión, se manifestaba en los centros de encierro y aislamiento, comprendiendo a estos como espacios disciplinarios, los cuales, eventualmente, en algún momento histórico entrarían en una crisis. Siguiendo la misma línea establecida por Foucault, Giles Deleuze muestra cómo el cambio de sociedad ya ocurrió:

Todos los centros de encierro atraviesan una crisis generalizada: cárcel, hospital, fábrica, escuela, familia[...] a un plazo más o menos largo, estas instituciones están acabadas [...] Solamente se pretende gestionar su agonía y mantener a la gente ocupada mientras se instalan esas nuevas fuerzas que ya están llamando a nuestras puertas. Se trata de las sociedades de control, que están sustituyendo a las disciplinarias [...] formas ultrarrápidas que adopta el control "al aire libre" y que reemplazan a las antiguas disciplinas que actuaban en el período de los sistemas cerrados (2006: 278).

A partir de lo señalado por Deleuze es posible comprender la relevancia que ha adquirido el fútbol, tomando distancia del resto de los deportes-espectáculos, tanto para los Estados como para el mercado, ya no solo siendo explicado como un fenómeno social masivo por su popularidad, sino como un fenómeno complejo ad hoc al paradigma imperante en la
Sociedad de Control. Los estadios fungen como espacios de tensión, ya no solo por la disputa en la producción de subjetividades, sino también como centro de estímulo, de posicionamiento social y de control del mercadeo.

El nuevo espectador que necesita la Sociedad de Control es aquel acorde a los tiempos actuales donde el neoliberalismo se ha masificado a escala global: este nuevo sujeto es el consumidor, cuyas principales características son: tener acceso constante y frecuente a los diferentes medios de masas; usuario acérrimo de las redes sociales; poseer una capacidad de endeudamiento acreditada por alguna agencia financiera que le permita no solo asistir a los encuentros deportivos, sino también comprar todo lo relacionado con clubes y selecciones, tanto nacionales como extranjeras. Este consumidor, híbrido y complejo, reemplaza al hincha, al barrista, e incluso al socio: hoy en día este "nuevo vínculo" se traduce en la figura del abonado, sujeto carente de voz y voto dentro de los clubes de fútbol, pero con su boleto de entrada asegurado. Foucault, en Vigilar y castigar describe el proceso por el cual avanza el sistema de sujeción del individuo, de la sociedad disciplinaria a la de control.

Permite, a la vez, la caracterización del individuo como individuo, y la ordenación de una multiplicidad dada. Es la condición primera para el control y el uso de un conjunto de elementos distintos: la base para una microfísica de un poder que se podría llamar "celular" (Foucault, 2002b: 137).

Sin embargo, este "derecho" que se le otorga al abonado, puede ser revocado en el momento en el que el organizador o sus asociados lo consideren pertinente. Esta modalidad insta a que el asistente abonado adopte una conducta normalizada, es decir, se autocontrole de acuerdo con los estándares conductuales preestablecidos.

En Europa se ha hecho más evidente la instauración del Estado de Excepción y su asimilación por parte de la sociedad, especialmente en 
el caso del fútbol. Los hinchas riesgosos han aceptado las nuevas reglas del juego, donde el empadronamiento como forma de control se ha asumido como única instancia que permite la asistencia a los encuentros deportivos; sin embargo, esta medida se traduce, a su vez, en una medida de exclusión, donde se identifica e individualiza al asistente que exhiba anomalías. El caso latinoamericano, como lo hemos visto, ha sido bastante dispar en su desplazamiento hacia la sociedad del control, siendo Chile a partir del 2011 el pionero, y manifestándose de forma masiva en Brasil antes y durante su mundial.

Las condiciones de acceso exigidas hoy en día para ingresar a un estadio dan cuenta de la exclusividad que ha adoptado el deporte. Exclusividad en términos de exclusión/ inclusión: se deberá cumplir lo exigido y asumir positivamente todos los procedimientos a los que se le someta, para mantener la conducta a raya. De no cumplirlo, aunque sea parcialmente, se corre el riesgo de quedar momentánea o permanentemente excluido.

Félix Guattari imaginaba una ciudad en la cual cada uno podía salir de su apartamento, de su casa o de su barrio gracias a su tarjeta electrónica (dividual) mediante la que iba levantando barreras; pero podría haber días u horas en los que la tarjeta fuera rechazada; lo que importa no es la barrera, sino el ordenador que señala la posición, lícita o ilícita, y produce una modulación universal (Deleuze, 2006: 284).

La sociedad de control ha avanzado y construido un nuevo tipo ideal de espectador transversal, en cuanto a todo tipo de eventos: ya no es el espectador culto, silente y crítico de la sociedad disciplinaria, hoy es consumidor apasionado, pero normalizado; es decir, consciente de que se "debe" autocontrolar y seguir la conducta normalizada que se le demanda. El consumidor del fútbol, como mencionamos, hoy no es el socio del club, es el abonado, no posee derechos ni está facultado para demandarlos, sus competencias se limitan a lo establecido en la relación clientilizada.

\section{El fútbol como dispositivo en el estado de excepción}

En este punto es necesario esclarecer estos dos conceptos para explicar la relación que tienen con el fútbol profesional. Dispositivo y estado de excepción han sido abordados por autores como Giorgio Agamben, Gilles Deleuze, Michel Foucault, Walter Benjamin y Carl Shimitt.

En cuanto a dispositivos nos remitiremos a la noción trabajada por Deleuze y Agamben, a partir de sus lecturas sobre Foucault; específicamente, cuando este último inicia sus indagaciones sobre la gubernamentalidad (el gobierno de los hombres) encontramos la siguiente explicación, que resume la función del dispositivo:

El dispositivo mismo es la red que tendemos entre estos elementos. [...] Por dispositivo entiendo una suerte, diríamos, de formación que, en un momento dado, ha tenido por función mayoritaria responder a una urgencia. De este modo, el dispositivo tiene una función estratégica dominante (Foucault, 2002: 229).

Entonces, dispositivo son todos aquellos lineamientos, que tienen como función orientar la conducta, normalizarla, corregirla, por tanto, su injerencia no solamente es explícita, sino también interna: penetra los cuerpos buscando establecerse en ellos, coaccionar desde adentro.

Pertenecemos a ciertos dispositivos y obramos en ellos. La novedad de unos dispositivos con respecto a los anteriores es lo que llamamos su actualidad, nuestra actualidad. Lo nuevo es lo actual: no es lo que somos sino más bien lo que vamos siendo, lo que llegamos a ser, es decir, lo otro, nuestra diferente evolución (Deleuze, 1990: 159).

Sin embargo, la sola noción de dispositivo no nos permite comprender cómo el fútbol se ha transformado en esto, en una fuente de lineamientos que normaliza a los sujetos, que des-subjetiviza en tanto que subjetiviza. De ser así, el poder del dispositivo, su capacidad 
de incidir en los cuerpos en instancias vacías, es decir, en un contexto neutro, inocuo, no logra ser ejercido. La Sociedad de Control funciona con base en dispositivos: estos crean o destruyen sujetos, los cuales actúan en pro de la normalización.

Consideremosquelos dispositivos, a simplevista, siempre surgen en contextos circunstanciales, como respuestas a situaciones anormales, desviadas, relacionadas con la a-legalidad; es decir, donde no es posible contemplar un "algo" que está contra la ley, sino más bien un "algo" fuera de la ley, mas no como excluido, sino como no contemplado o invisibilizado. La visibilización, así, es la función principal del dispositivo, lo que permite expandir el sistema referencial de inclusión/exclusión.

El marco legislativo, sin embargo, dentro de sus limitaciones no puede aprehender lo que no contempla, lo que le es invisible, por estar fuera de este; por tanto, su visibilidad solo es posible por entes que se encuentren en la misma situación que el objeto a visibilizar; en otras palabras, es un ente implantado fuera de la misma ley. Es por esta razón que, en concordancia con Agamben, el contexto en el que nos situamos es el de un estado de excepción, donde lo fuera de la norma es la norma misma:

El estado de excepción no es una dictadura (constitucional o inconstitucional, comisarial o soberana), sino un espacio vacío de derecho, una zona de anomia en la cual todas las determinaciones jurídicas " $y$, sobre todo, la distinción misma entre público y privado" son desactivadas (Agamben, 2010: 99).

El fútbol, por tanto, es un dispositivo que, no siendo contemplado por la ley misma, establece por sí mismo los parámetros para la ampliación de su cobertura de inclusión/exclusión, por una parte, y por otra, el perfeccionamiento en el control del sistema referencial; es decir, las medidas que regulan el acceso o el rechazo del sistema mismo.
Sobre el estado de excepción se sitúa el concepto soberano de Schmitt, pero también existe la posibilidad, según Agamben, de que los sujetos, en tanto abyectos, se sitúen fuera del marco epistémico establecido, construyendo los cimientos de una posible y necesaria revolución. Los puntos de fuga del sistema implican una subsistencia fuera de este y desde parámetros diferentes, pero que están en paralelo. La figura del barrista hoy es contemplada, incluso sobrevisibilizada: lo vemos en el mercadeo que rodea al deporte; sin embargo, como tal, ha sido excluido, formalmente, de la actividad misma; su figura original ha sido trastocada, en un contexto donde hoy contempla los márgenes de lo que ha sido normalizado, un ferviente consumidor de fútbol.

\section{Conclusiones}

Los mundiales de fútbol del presente siglo han evidenciado lo que Agamben ya había anunciado desde el período de entre guerras: la instauración del estado de excepción como la norma vigente, el cual absorbe terrenos que históricamente había obviado, olvidado o excluido. La explosión del fútbol como una gran empresa, tomando distancia del resto de los deportes, sirvió como aliado perfecto para expandir la sociedad de control.

Entender al fútbol como dispositivo nos permite seguir comprendiendo el actuar biopolítico de la sociedad de control: una reconfiguración de subjetividades que busca situarlas en el marco de inclusión/exclusión. Este contexto de control impulsa a los mismos sujetos a demandar el perfeccionamiento del sistema, pero al no ser un sistema disciplinario, ilusiona con la libertad, solo en la medida en que los sujetos se autocontrolen y se adapten al sistema y exijan al resto de pares su normalización.

Los acontecimientos analizados evidencian las instancias tras las cuales se ha ido perfeccionando el sistema de control, circunstancias que sirvieron como fundamento, instauración y justificación para su rápida masificación. 
Finalmente, vemos cómo hay una zona gris que podemos denominar como fuera de la ley, en la cual si bien el Estado suele situarse por sobre esta (sin domarla del todo), también vemos cómo es posible ingresar a esta y así construir las condiciones para gestar una lucha ante la dominación. En cuanto a los sujetos, las barras, y sobre todo, la figura del barrista se puede considerar como un punto de fuga dentro de la concepción actual de fútbol, donde los marcos normativos han intentado excluirle y combatirlo; sin embargo, es un fenómeno que ha seguido creciendo y expandiéndose de forma (des) controlada. Los sectores de la barra dentro de los estadios se han convertido en verdaderas trincheras que muestran cierta relación de tensión, en el marco de la producción de subjetividades.

\section{Referencias}

Libros y artículos en revistas académicas

Agamben, G. (2010). Estado de Excepción (Cuarta ed.). Buenos Aires: Adriana Hidalgo Editora.

Agamben, G. (2011). “Qué es un dispositivo?", Sociológica, núm. 73, pp. 249-264.

Deleuze, G. (1990). “Qué es un dispositivo?”, en G. Deleuze, A. Glucksmann, M. Frank y E. Balbier, Michel Foucault. Filósofo, pp. 155163, Barcelona: Gedisea.

Deleuze, G. (2006). Conversaciones 1972-1990. Valencia: Pre-Textos.

Foucault, M. (2002a). Dichos y escritos (vol. III). Madrid: Editorial Nacional.

Foucault, M. (2002b). Vigilary castigar: nacimiento de la prisión (primera ed.). Buenos Aires: Siglo XXI Editores.

Gidddens, A. (2001). Sociología. Madrid: Alianza Editorial.
Revistas, periódicos y páginas web oficiales y de medios de comunicación

BBC Mundo (2010). "Sudáfrica 2010: reportan a 'barrabravas argentinos' ", 16 de junio, recuperado de La Tercera, versión on line: http://www.latercera.com/ noticia/mundo/bbc-mundo/cultura-ysociedad/2010/06/1437-268615-9-bbcsudafrica-2010-deportan-a-barrabravasargentinos.shtml

El Universo. (2010). "Barras bravas expulsados de Sudáfrica", 16 de junio, http://www. eluniverso.com/2010/06/07/1/1372/ barrabravas-expulsados-sudafrica.html

Fernández, B. (2014). “El prontuario de la 'Marea Roja' en Brasil 2014", El Mostrador, 26 de junio, http://www.elmostrador.cl/ pais/2014/06/26/el-prontuario-de-lamarea-roja-en-brasil-2014/

Gobierno de Chile (2012). "Cuenta Pública Sectorial 2012", Ministerio del Interior y Seguridad Pública. junio:

h t t p : / / w w w. in terior. gob.cl/m ed i a / $2014 / 06 /$ CuentaPublicaSectorial2012.pdf

González, M. (2014). "La ANFP veta a hinchas chilenos que invadieron el Maracaná", La Cuarta. El diario popular, 20 de junio, recuperado de. http://www.lacuarta. com/noticias/deportiva/2014/06/64169766-9-la-anfp-veta-a-hinchaschilenos-que-invadieron-el-maracana. shtml

La Tercera. (2010). "Deportan a los barrabravas argentinos retenidos en Sudáfrica", 23 de junio http://www.latercera.com/noticia/ deportes/2010/06/656-270473-9deportan-a-los-barrabravas-argentinosretenidos-en-sudafrica.shtml

Radio Cooperativa. (2013). “Dilma Rousseff y Joseph Blatter fueron abucheados en 
la Copa Confederaciones", 15 de junio, http://www.cooperativa.cl/noticias/ deportes/futbol/copa-confederaciones/ dilma-rousseff-y-joseph-blatter-fueronabucheados-en-la-copa-confederacion es/2013-06-15/161858.html
ZGS. (2010,). “Polémica en Argentina por viaje de 'barrabravas' al Mundial de Sudáfrica", Hola Ciudad!, 3 de junio, http://www.holaciudad.com/polemicaargentina-viaje-barrabravas-al-mundialsudafrica-n84196 